between YORA and LORA were not significantly different at initial presentation (table 1). The ultrasound characteristics differed between these two groups. LORA patients were more likely to have shoulder biceps tendon tenosynovitis (GS; $p=0.026, P D ; 0.037$ ), elbow joint synovitis (GS; $p=0.010, P D ; p=0.037$ ), MCP1 (PD; $p=0.032$ ) and MCP5 (GS; $p=0.035$ ) synovitis, compared to YORA patient. YORA patients were more likely to have MTP synovitis (GS; $p=0.013$ ) compared to LORA patients.

Conclusions: This is the first study to describe the difference of both clinical and sonographic inflammation of YORA and LORA in recent-onset DMARD-naïve RA patients in a longitudinal study. There are differences in US-detected joint and tendon inflammation despite similarities in clinical characteristics. The prognostic value of the differences in US pathology between these two groups should be further explored.

Disclosure of Interest: None declared

DOI: 10.1136/annrheumdis-2018-eular.5805

\section{OP0153 RESOLUTION OF SYMPTOMS OF PATIENTS WITH CLINICALLY SUSPECT ARTHRALGIA IS PARALLELED BY DISAPPEARANCE OF MRI-DETECTED SUBCLINICAL INFLAMMATION; A LONGITUDINAL STUDY}

R.M. Ten Brinck, D.M. Boeters, H.W. van Steenbergen, A.H. van der Helm- van Mil. Rheumatology, LEIDEN UNIVERSITY MEDICAL CENTRE, Leiden, Netherlands

Background: It is known that MRI-detected subclinical inflammation in patients with Clinically Suspect Arthralgia (CSA) precedes the development of clinically evident Inflammatory Arthritis (IA). However, there are no longitudinal studies in CSA patients that did not progress to IA. The course of joint symptoms in these patients is unknown. Furthermore, it is unknown if subclinical inflammation resolves spontaneously in parallel with disappearance of joint symptoms.

Objectives: To determine the course of joint symptoms and of MRI-detected subclinical joint inflammation, and mutual time relationships, in patients that presented with CSA but did not progress to IA during 2 year follow-up.

Methods: Between April 2012-April 2014, 149 patients were included in the CSA cohort. These patients had no clinically evident arthritis but recent-onset $(<1$ year) arthralgia of small joints that was clinically considered at risk for IA by the rheumatologist. None of the patients were treated with DMARDs. Patients underwent MRI at baseline and when arthritis developed or after 2 years (when follow-up ended) The patients that did not progress to IA within 2 years and had serial MRIs, as well as longitudinal clinical data and questionnaires (e.g. the $\mathrm{HAQ}$ ), were studied $(n=60)$. 1.5T MRIs of wrist, MCP2-5, and MTP1-5 joints were scored for synovitis, tenosynovitis and bone marrow oedema (BME) (summed in the total MRI inflammation score) according to RAMRIS, blinded for order in time and clinical information. Paired t-tests were used.

Results: After 2 year follow-up, 20 patients (33\%) had complete resolution of symptoms whereas 40 patients $(66 \%)$ did not. The remaining symptoms in the latter group were diagnosed as: persistent inflammatory arthralgia $(n=26)$, osteoarthritis $(n=6)$ and tendinomyalgia $(n=8)$. In the total group, the mean total MRIinflammation score decreased from 3.2 at baseline to 2.5 at 2 years $(p=0.046)$. When the patients with persistent symptoms were studied, there were no significant changes in MRI-inflammation scores. Analysing the patients with resolution of symptoms separately, the total MRI-inflammation score decreased significantly ( 3.3 to $2.3 ; p=0.019$ ). Also the total synovitis and tenosynovitis scores decreased significantly ( 1.3 to 0.82 and 0.83 to 0.30 , respectively; $p=0.043$ and $p=0.029$ ), whereas BME scores remained unchanged (1.2 and 1.2; $p=0.87$ ). After 2 year follow-up the absolute MR-scores of these patients were compared to data from age-matched symptom-free controls; no differences were found for synovitis, sug gesting normalisation. Physical functioning, measured with $\mathrm{HAQ}$, remained similar in patients with persistent symptoms ( 0.56 to $0.51 ; p=0.61)$, whereas HAQ normalised in patients with resolution of symptoms (mean score of 0.15 at 2 years, $\mathrm{p}=0.053$ compared to baseline)

Conclusions: Approximately one-third of the patients that presented with CSA and that did not convert to IA had resolution of symptoms, this was paralleled by normalisation of MRI-detected inflammation as well as physical functioning. This is the first study showing that subclinical inflammation in individuals at risk for developing RA can resolve spontaneously. The data suggest that subclinical inflammation was causally related to joint pain.

Disclosure of Interest: None declared

DOI: 10.1136/annrheumdis-2018-eular.3436

\section{OP0154 \\ PROSPECTIVE OBSERVATIONAL STUDY TO EVALUATE THE USE OF MUSCULOSKELETAL ULTRASONOGRAPHY TO IMPROVE RHEUMATOID ARTHRITIS MANAGEMENT: INTERIM ANALYSIS OF THE ECHO STUDY}

$\underline{M ~ S t e i n ~}^{1,2}$, E. Rampakakis ${ }^{3}$, J.S. Sampalis ${ }^{2,3} .{ }^{1}$ McGil University, ${ }^{2}$ Canadian Rheumatology Ultrasonography Society (CRUS), Montreal; ${ }^{3}$ JSS Medical Research, St. Laurent, Canada

Background: Musculoskeletal Ultrasound (MSUS) has been shown to be supe rior to clinical examination in the detection of synovitis in patients with Rheumatoid Arthritis (RA), and can be used to improve diagnostic accuracy and potentially monitor disease changes in order to make treatment decisions aimed at optimising patient care. Since the creation of the Canadian Rheumatology Ultrasonography Society (CRUS) in 2010, an increasing number of rheumatologists has been trained in the use of MSUS.

Objectives: The overall study objective is to compare the effectiveness of MSUS to Routine Care $(\mathrm{RC})$ as a disease management tool in patients with moderate-tosevere RA for whom a change in treatment is indicated. In addition, the predictive power of MSUS assessments has been assessed here.

Methods: 'Echo' is a prospective two-cohort, quasi-experimental study of patients diagnosed with active moderate-to-severe RA managed either with MSUS (within CRUS) or as per RC. To be eligible for the study patients must require a change in treatment as per the judgment of the treating physician. Patients are followed for 1 year with assessments at baseline, 3, 6, 9, and 12 months. Key outcome measures of interest include CDAI LDA/Remission, DAS 28 LDA/Remission, patient satisfaction (TSQM) and patient perception of participation in disease management (PAM-13).

Results: A total of 383 patients (71.5\% female) with a mean (SD) age of 58.7 (11.7) years and disease duration of 7.0 (10.0) years were enrolled, without any significant differences between treatment groups.

At baseline, a greater proportion of patients in the MSUS group were treated with a biologic DMARD (bDMARD; $50.3 \%$ vs $35.8 \%, p=0.004$ ); patients in the RC group were more likely to be treated with a non-biologic DMARD (nbDMARD; $84.2 \%$ vs $91.5 \%, p=0.027$ ). Over time, a comparable proportion of patients in the two groups started/switched a bDMARD $(21.6 \%$ vs $15.6 \%, \mathrm{p}=0.126)$ or added/ switched a nbDMARD $(18.7 \%$ vs $23.6 \%, p=0.248)$. The overall number of treatment modifications was also similar between groups ( 3.0 vs $2.7, p=0.236$ ).

Upon adjusting for age, gender, previous bDMARD treatment, and baseline parameter level, no differences between the two treatment groups with respect to CDAI LDA/Remission, DAS-28 LDA/Remission, and TSQM score were observed during follow-up. However, the PAM-13 score was significantly higher in the MSUS group (69.6 vs $64.2, p=0.02$ )

In the MSUS group, higher total US erosion score at baseline was associated with a lower rate of CDAI LDA at 12 months $(O R=0.86 ; p=0.047)$; higher total $P D$ synovitis score at baseline was associated with a lower rate of CDAI LDA at 6 months $(\mathrm{OR}=0.90 ; \mathrm{p}=0.010)$, and; higher total synovitis GREY scale at baseline was associated with lower rates of DAS28 LDA $(O R=0.93 ; p=0.026)$ and DAS28 remission $(\mathrm{OR}=0.94 ; \mathrm{p}=0.061)$ at 6 months.

Conclusions: MSUS assessments can be useful predictors of future disease remission in patients with RA. MSUS may be associated with increased patien perception of participation in disease management and patient activation.

Acknowledgements: The Sponsors of this investigator-initiated study are the Canadian Rheumatology Ultrasonography Society (CRUS) and JSS Medical Research (in-kind support). The study is supported by an unrestricted grant by AbbVie.

Disclosure of Interest: None declared

DOI: 10.1136/annrheumdis-2018-eular.3563

\section{OP0155 ULTRASOUND AS AN OUTCOME MEASUREMENT TOOL FOR OPTIMISEDMONITORING OF GOUT. VALIDATION OF THE OMERACT ULTRASOUND DEFINITIONS OF GOUT ELEMENTARY LESIONS}

S.N. Christiansen, M. Østergaard, O. Slot, L. Terslev. Center for Rheumatology and Spine Diseases, Copenhagen Center for Arthritis Research, Rigshospitalet, Glostrup, Glostrup, Denmark

Objectives: To evaluate ultrasound (US) as an outcome measurement instrument for monitoring gout patients during urate lowering therapy using the OMERACT US Working Group's 2015 definitions of US elementary lesions in gout.

Methods: US examination (28 joints, 26 tendons) were performed in patients with microscopically verified gout who either initiated or increased urate lowering therapy. Joints and tendons were evaluated for the four OMERACT elementary lesions of gout (Double contour, Tophus, Aggregates and Erosions). Furthermore subcutaneous ( $\mathrm{SC}$ ) oedema was registered and synovitis was graded by grey scale (GS) and colour Doppler (CD) (both graded 0-3). A sum score was 
calculated for each component for each patient (table 1). Patient Reported Outcomes (PROs) regarding pain (visual analogue scale), numbers of attacks within the last 3 months and physical function (Health Assessment Questionnaire) were obtained, as were $\mathrm{C}$-reactive protein (CRP), p-urate and clinical joint examination All examinations were repeated after $3(n=29)$ and 6 months $(n=15$, follow-up still ongoing) and changes in scores were evaluated using Wilcoxon-Pratt signedrank test.

Results: 29 patients (28 males, 1 female), mean age of $68(39-89)$ years were included. US showed a numerical, but statistically non-significant $(p=0.13)$, decline in DC count from baseline to 3 months' follow up, while at 6 months a statistically significant decline was observed $(p=0.033)$. The tophus count decreased non-significantly at both 3 and 6 months' follow up, whereas the aggregate and erosion counts by large were unchanged. GS synovitis showed a statistically nonsignificant decrease at follow ups, whereas CD synovitis and SC oedema counts declined significantly at 3 months' follow up ( $p=0.033$ and 0.044 , respectively).

$\mathrm{P}$-urate levels decreased statistically significant from baseline to both 3 and 6 months' follow-up (both p-values $<0.001$ ), as did clinical markers such as CRP, joint evaluation, pain and attack frequency.

Abstract OP0155 - Table 1 Course of US, biochemical and clinical variables during urate lowering therapy

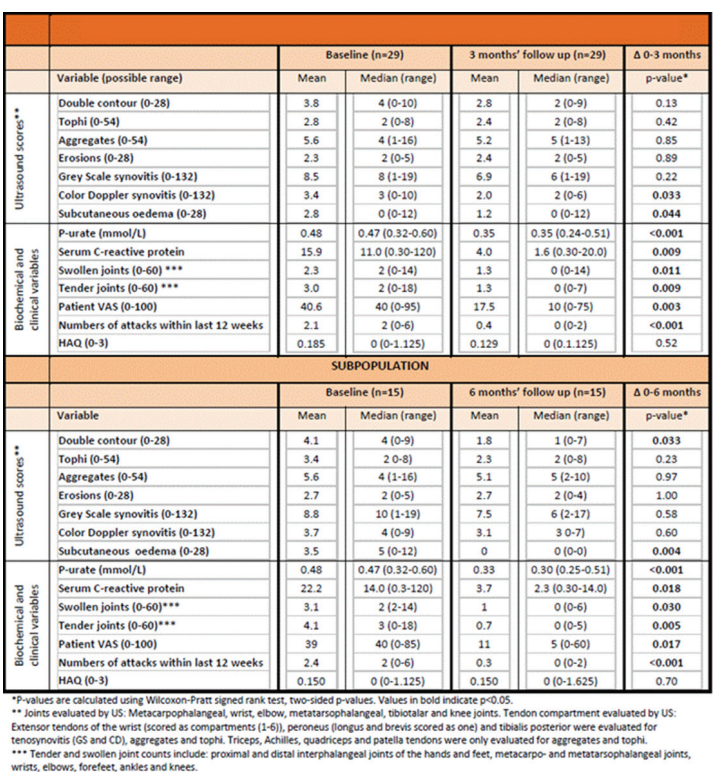

Conclusions: Of the four OMERACT US elementary gout lesions only DC count showed a statistically significant decrease as a response to 6 months of urate lowering therapy. The number of tophi had decreased at both 3 and 6 months' follow up, but not statistically significant. Aggregates and erosions count did not markedly respond to the 6 month treatment.

The study indicates that US assessing the OMERACT elementary lesions, particularly DC, is a feasible tool for monitoring gout lesions. However, a follow-up of at least 6 months may be needed to detect change of crystal deposits, as reflected by $\mathrm{DC}$, and presumably an even longer follow-up period is needed to evaluate more massive deposits as tophi.

Disclosure of Interest: None declared

DOI: 10.1136/annrheumdis-2018-eular.2209

\section{OP0156 SIMPLE ASSESSMENT OF CONVENTIONAL 18F-FDG PET/CT ACCURATELY DIAGNOSES CRANIAL ARTERITIS IN GLUCOCORTICOID-NAÏVE GCA PATIENTS: A CASE-CONTROL STUDY}

B.D Nielsen ${ }^{1,2}$, I.T. Hansen ${ }^{3,4}$, A. Haraldsen ${ }^{5}$, S. Kramer ${ }^{5}$, K.K. Keller ${ }^{1}$, P. Therkildsen ${ }^{1}$, E.-M. Hauge ${ }^{1}$, L.C. Gormsen ${ }^{1} .{ }^{1}$ Rheumathology; ${ }^{2}$ Clinical medicine; ${ }^{3}$ Rheumatology, Aarhus University Hospital; ${ }^{4}$ Clinical medicine, Aarhus University;

${ }^{5}$ Nuclear Medicine and PET, Aarhus University Hospital, Aarhus, Denmark

Background: Although older studies argue that fluorine-18-fluorodeoxyglucose (FDG) positron emissions tomography (PET)/CT cannot demonstrate inflammation in cranial arteries the spatial resolution of modern PET systems have greatly improved allowing for more precise diagnostics of small structures. FDG PET/CT is widely used to diagnose large-vessel (LV) giant cell arteritis (GCA). Recognising FDG uptake in cranial arteries potentially adds to FDG PET/CTs diagnostic accuracy for GCA.

Objectives: To evaluate the diagnostic accuracy of conventional FDG PET/CT of the cranial arteries in GCA.

Methods: In a cohort of consecutively included glucocorticoid-naïve patients sus pected of new-onset GCA, patients full-filling 1990 ACR criteria for GCA were identified. Conventional FDG PET/CT and clinical assessment was performed before treatment. Controls were age- and sex-matched patients with malignant melanoma (MM) who had a metastatic-disease-free follow-up FDG PET/CT $\geq 6$ months after MM resection.

All PET images were evenly cropped to include only head and neck. Images were randomly assessed by 2 nuclear medicine physicians (10 years experience) blinded to clinical symptoms and findings. Training included review of 5 GCA-PET examinations (not part of cohort). Temporal (TA), maxillary (MA) and vertebra (VA) arteries were visually scored bilaterally. Arterial FDG uptake above surrounding tissue was considered indicative of inflammation and graded low or high. If disagreement between readers occurred, final score was settled by an expert nuclear medicine physician.

Student t test was used for quantitative data. Inter-reader agreement was evaluated by Cohens weighted kappa (disagreement on diagnosis weighted 0 , disagreement on FDG uptake intensity weighted 0.2).

Results: A total of 44 patients and 44 controls were identified. In both case and control group, the mean age was 69 years $(p=0.45)$ and 25/44 were women. Large-vessel involvement was seen in 39/42 patients, and 35/42 were temporal artery biopsy positive. GCA patients' median global assessment of disease activity was 8 (IQR: $5-10)$ and median CRP was $70(95 \% \mathrm{Cl}: 58 ; 85) \mathrm{mg} / \mathrm{L}$.

Considering only FDG uptake in TA and/or MA, diagnostic sensitivity and specificity was $66 \%$ (95\% Cl: $50 \%-80 \%)$ and $100 \%(95 \% \mathrm{Cl}: 92 \%-100 \%)$. Including VA sensitivity increased to $86 \%(95 \% \mathrm{Cl}: 73 \%-95 \%)$ and specificity remained high, $98 \%$ (95\% Cl: $88 \%$ to $100 \%$ ). Cohens weighted kappa was 0.82 (agreement $93 \%$, $\mathrm{p}=0.000$ ) in a per segment analysis and kappa was 0.84 (agreement $92 \%$, $\mathrm{p}=0.000)$ in diagnosis.

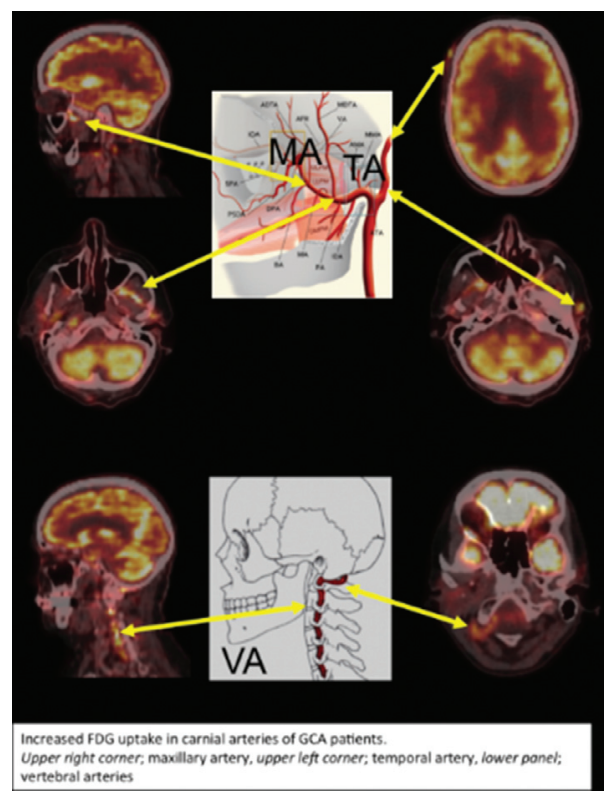

Absatrct OP0156 - Figure 1 FDG PET/CT of cranial arteries in patients with giant cell arteritis

Conclusions: Inter-reader agreement on FDG uptake in cranial arteries is almost perfect, and cranial arteritis in glucocorticoid-naïve GCA patients can be readily and accurately diagnosed by conventional FDG PET/CT. The high diagnostic specificity suggests that TAB can be avoided in patients with FDG uptake in cranial arteries. Moreover, FDG PET/CT performed in patients with suspected vasculitis should always include head and neck.

Disclosure of Interest: None declared

DOI: 10.1136/annrheumdis-2018-eular.1338 